\title{
LRS Bianchi-I Anisotropic Cosmological Model with Dominance of Dark Energy
}

\author{
Anil Kumar Yadav ${ }^{\dagger}$ and Bijan Saha ${ }^{\ddagger}$ \\ ${ }^{\dagger}$ Department of Physics, Anand Engineering College, Keetham, Agra-282 007, India \\ $\dagger$ E-mail: abanilyadav@yahoo.co.in \\ ${ }^{\ddagger}$ Laboratory of Information Technologies, Joint Institute for Nuclear Research \\ Dubna - 141980, Russia \\ ${ }^{\ddagger}$ E-mail : bijan@jinr.ru ${ }^{\ddagger}$ URL : http://bijansaha.narod.ru
}

\begin{abstract}
The present study deals with spatially homogeneous and anisotropic locally rotationally symmetric (LRS) Bianchi type I cosmological model with dominance of dark energy. To get the deterministic model of Universe, we assume that the shear scalar $(\sigma)$ in the model is proportional to expansion scalar $(\theta)$. This condition leads to $A=B^{n}$, where $A, B$ are metric potential and $n$ is positive constant. It has been found that the anisotropic distribution of dark energy leads to the present accelerated expansion of Universe. The physical behavior of the Universe has been discussed in detail.
\end{abstract}

Keywords: LRS Bianchi type I Universe, dark energy and distance modulus curve PACS number: $98.80 . \mathrm{Cq}, 04.20 .-\mathrm{q}, 04.20 . \mathrm{Jb}$

\section{Introduction}

The discovery of the accelerated mode of expansion of the Universe stands as a major breakthrough of the observational cosmology. Survey of cosmological distant type Ia supernovae (SNe Ia; Riess et al 1998; Perlmutter et al 1999) indicated the presence of a new unaccounted-for Dark energy (DE) that opposes the self-attractions of matter and causes the expansion of Universe to accelerate. This acceleration is realized with negative pressure and positive energy density that violate the strong energy condition. This violation gives a reverse gravitational effect. Due to this effect, the Universe gets a jerk and the transition from the earlier deceleration phase to the recent acceleration phase takes place (Caldwell et al. 2006). The cause of this sudden transition and the source of accelerated expansion are still unknown. The state of the art in cosmology has led to the following present distribution of the energy densities of the Universe: $4 \%$ for baryonic matter, $23 \%$ for non baryonic dark matter and $73 \%$ so-called DE (Spergel et al. 2007).

The isotropy of the cosmic microwave background (CMB) radiation, first seen by the cosmic background explorer (COBE) satellite (Smoot et al. 1992) and then reinforced by the Wilkinson Microwave Anisotropy Probe (WMAP) data (Hinshaw et al. 2003), together with the assumption that we are not in spacial position in the Universe, underlines the cosmological principles, according to which we live in a homogeneous and isotropic Universe described by a FRW line-element. Tiny deviation from perfect isotropy at the level of $10^{-5}$, have also been reported by Bennett et al (1996) and thereafter confirmed by high resolution WMAP data. The observed CMB anisotropy spectrum is in impressive agreement with the predictions of $\Lambda$ CDM model. Koivisto and Mota (2008a, 2008b) proposed the mechanism of DE with anisotropic equation of state (EoS) parameter which is very attractive because cosmic anisotropy originates from the actual dominant component of the Universe and then could be directly tested, for example, by either observations of the magnitude and redshift of type Ia supernovae or cosmic parallax effects of the distance source. DE has been conventionally characterized by the equation of state $(\mathrm{EoS})$ parameter $\omega^{(d e)}=p^{(d e)} / \rho^{(d e)}$ which is not necessarily constant. The simplest DE candidate is the vacuum energy $(\omega=-1)$, which is argued to be equivalent to the cosmological constant $(\Lambda)$ (Martins, 2002). However, it is well known, there are two difficulties arising from the cosmological constant scenario, namely the two famous cosmological constant problems - the fine tuning and the cosmic coincidence one. An alternative proposal is the concept of dynamical DE. Such a scenario is often realized by some scalar field mechanism and suggests that the energy form with negative pressure is provided by a scalar field evolving under a properly 
constructed potential. So far, a large class of scalar-field DE models have been studied, including quintessence viz $\omega^{(d e)}>-1$ (Steinhardt et al 1999), phantom viz $\omega^{(d e)}<-1$ (Caldwell 2002) and quintom (that can across from phantom region to quintessence region). The quintom scenario of $\mathrm{DE}$ is designed to understand the nature of DE with $\omega^{(d e)}$ across -1 (Setare 2006). Recently, Cai et al (2010), Setare and Saridakis (2007, 2009b) have studied the DE models with EoS parameter across -1 which give a concrete theoretical justification for quintom paradigm. In addition, the other proposals on DE include interacting DE model (Setare 2007) and braneworld model (Setare and Saridakis 2009a) etc. By combining data from seven CMB experiments with large scale structure data, the Hubble parameter measurement from the Hubble space-telescope and luminosity measurements of SN Ia, Melchiorri et al (2003) demonstrated the bound on $\omega^{(d e)}$ to be $-1.38<\omega^{(d e)}<-0.82$ at $95 \%$ confidence level.

The simplest of anisotropic models are Bianchi type-I homogeneous models whose spatial sections are flat but the expansion or contraction rate are direction dependent. For studying the possible effects of anisotropy in the early Universe on present day observations many researchers (Huang 1990; Chimento et al. 1997; Lima and Troden 1996; Lima and Maia 1994; Pradhan and Singh 2004; Pradhan and Pandey 2006; Saha 2006a, 2006b) have investigated Bianchi type-I models from different point of view. The binary mixture of perfect fluid and DE has been studied for Bianchi type I (Saha 2005). Some Authors (Akarsu and Kilinc 2010; Yadav and Yadav 2011; Yadav et al 2011b; Kumar and Yadav 2011; Amirhashchi et al 2011 and recently Yadav 2011) have studied anisotropic DE models with constant deceleration parameter (DP).

In this paper, we considered minimally interacting perfect fluid and DE components with proportionality relation between shear scalar and expansion within the framework of LRS Bianchi-I space-time in general relativity. The paper is organized as follows: In section 2, the models and field equations have been presented. Section 3 deals with the exact solutions of field equations and physical behavior of the model. The statefinder and distance modulus curves are described in section 4 . Finally the results are discussed in section 5 .

\section{The Metric and Field Equations}

We consider the LRS Bianchi type I metric of the form

$$
d s^{2}=-d t^{2}+A^{2} d x^{2}+B^{2}\left(d y^{2}+d z^{2}\right),
$$

where, A and B are functions of $t$ only. This ensures that the model is spatially homogeneous.

The Einstein's field equations in case of a mixture of perfect fluid and DE components, in the units $8 \pi G=$ $c=1$, read as

$$
R_{j}^{i}-\frac{1}{2} g_{j}^{i} R=-T_{j}^{i}
$$

where $T_{j}^{i}=T_{j}^{(m) i}+T_{j}^{(d e) i}$ is the overall energy momentum tensor with $T_{i j}^{(m)}$ and $T_{i j}^{(d e)}$ as the energy momentum tensors of ordinary matter and $\mathrm{DE}$, respectively. These are given by

$$
T_{j}^{(m) i}=\operatorname{diag}\left[-\rho^{(m)}, p^{(m)}, p^{(m)}, p^{(m)}\right],=\operatorname{diag}\left[-1, \omega^{(m)}, \omega^{(m)}, \omega^{(m)}\right] \rho^{(m)}
$$

and

$$
T_{j}^{(d e) i}=\operatorname{diag}\left[-\rho^{(d e)}, p^{(d e)}, p^{(d e)}, p^{(d e)}\right]=\operatorname{diag}\left[-1, \omega^{(d e)}, \omega^{(d e)}, \omega^{(d e)}\right] \rho^{(d e)}
$$

where $\rho^{(m)}$ and $p^{(m)}$ are, respectively the energy density and pressure of the perfect fluid component or ordinary baryonic matter while $\omega^{(m)}=p^{(m)} / \rho^{(m)}$ is its EoS parameter. Similarly, $\rho^{(d e)}$ and $p^{(d e)}$ are, respectively the energy density and pressure of the DE component while $\omega^{(d e)}=p^{(d e)} / \rho^{(d e)}$ is the corresponding EoS parameter.

The Einstein's field equations (2) for the line-element (11) lead to the following system of equations

$$
\begin{gathered}
2 \frac{\ddot{B}}{B}+\frac{\dot{B}^{2}}{B^{2}}=-\omega^{(m)} \rho^{(m)}-\omega^{(d e)} \rho^{(d e)}, \\
\frac{\ddot{A}}{A}+\frac{\ddot{B}}{B}+\frac{\dot{A} \dot{B}}{A B}=-\omega^{(m)} \rho^{(m)}-\omega^{(d e)} \rho^{(d e)},
\end{gathered}
$$




$$
\frac{\dot{B}^{2}}{B^{2}}+2 \frac{\dot{A} \dot{B}}{A B}=\rho^{(m)}+\rho^{(d e)}
$$

The Bianchi identity $G_{; j}^{i j}=0$ yields

$$
\dot{\rho}^{(m)}+3\left(1+\omega^{(m)}\right) \rho^{(m)} H+\dot{\rho}^{(d e)}+3\left(1+\omega^{(d e)}\right) \rho^{(d e)} H=0,
$$

with $H$ being the mean Hubble parameter, which for LRS Bianchi I space-time can be defined as

$$
H=\frac{\dot{a}}{a}=\frac{1}{3}\left(\frac{\dot{A}}{A}+2 \frac{\dot{B}}{B}\right)
$$

Here, and in what follows, over-dots indicates differentiation with respect to $t$ and $a$ is the average scale factor of LRS Bianchi type I model:

$$
a=\left(A B^{2}\right)^{\frac{1}{3}}
$$

The spatial volume $(\mathrm{V})$ is given by

$$
V=a^{3}=A B^{2} .
$$

The expansion scalar $(\theta)$, shear scalar $(\sigma)$ and mean anisotropy parameter $\left(A_{m}\right)$ are defined as

$$
\begin{gathered}
\theta=3 H=\frac{\dot{A}}{A}+2 \frac{\dot{B}}{B}, \\
\sigma^{2}=\frac{1}{2}\left(\sum_{i=1}^{3} H_{i}^{2}-\frac{1}{3} \theta^{2}\right), \\
A_{m}=\frac{1}{3} \sum_{i=1}^{3}\left(\frac{H_{i}-H}{H}\right)^{2} .
\end{gathered}
$$

\section{Solutions of the Field Equations}

In order to solve the field equations completely, firstly we assume that the perfect fluid and DE components interact minimally. Therefore, the energy momentum tensors of the two sources may be conserved separately.

The energy conservation equation $T_{; j}^{(m)} i j=0$, of the perfect fluid leads to

$$
\dot{\rho}^{(m)}+3\left(1+\omega^{(m)}\right) \rho^{(m)} H=0,
$$

whereas the energy conservation equation $T_{; j}^{(d e) i j}=0$, of the DE component yields

$$
\dot{\rho}^{(d e)}+3\left(1+\omega^{(d e)}\right) \rho^{(d e)} H=0 .
$$

Following Akarsu and Kilinc (2010), we assume that the EoS parameter of the perfect fluid to be a constant, that is,

$$
\omega^{(m)}=\frac{p^{(m)}}{\rho^{(m)}}=\text { const. }
$$

while $\omega^{(d e)}$ has been allowed to be a function of time since the current cosmological data from SNIa, CMB and large scale structures mildly favor dynamically evolving DE crossing the phantom divide line (PDL) as discussed in Section 1.

Finally, we constrain, the system of equation with proportionality relation between shear $(\sigma)$ and expansion $(\theta)$. This condition leads to the following relation between the metric potentials

$$
A=B^{n}
$$

where $n$ is positive constant. For anisotropic model $n \neq 1$.

Equations (6), (7) and (18) lead to

$$
\frac{\ddot{B}}{B}+(n+1) \frac{\dot{B}^{2}}{B^{2}}=0
$$


The solution of equation (20) is given by

$$
B=\left(k_{1} t+k_{0}\right)^{\frac{1}{n+2}},
$$

Where $k_{0}$ and $k_{1}$ are the constants of integration.

From equations (18) and (20), we obtain

$$
A=\left(k_{1} t+k_{0}\right)^{\frac{n}{n+2}} .
$$

The rate of expansion in the direction of $x, y$ and $z$ are given by

$$
\begin{gathered}
H_{x}=\frac{\dot{A}}{A}=\frac{n k_{1}}{(n+2)} \frac{1}{\left(k_{1} t+k_{0}\right)}, \\
H_{y}=H_{z}=\frac{\dot{B}}{B}=\frac{k_{1}}{(n+2)} \frac{1}{\left(k_{1} t+k_{0}\right)} .
\end{gathered}
$$

The mean Hubble's parameter $(H)$, expansion scalar $(\theta)$ and shear scalar $(\sigma)$ are given by

$$
\begin{gathered}
H=\frac{k_{1}}{3\left(k_{1} t+k_{0}\right)}, \\
\theta=\frac{k_{1}}{\left(k_{1} t+k_{0}\right)}, \\
\sigma^{2}=\frac{(n-1)^{2} k_{1}^{2}}{3(n+2)^{2}} \frac{1}{\left(k_{1} t+k_{0}\right)^{2}} .
\end{gathered}
$$

The spatial volume $(\mathrm{V})$, mean anisotropy parameter $\left(A_{m}\right)$ and DP $(q)$ are found to be

$$
\begin{gathered}
V=\left(k_{1} t+k_{0}\right) \\
A_{m}=\frac{2(n-1)^{2}}{(n+2)^{2}} \\
q=\frac{d}{d t}\left(\frac{1}{H}\right)-1=2 .
\end{gathered}
$$

From equations (25) and (26), we obtain

$$
\frac{\sigma}{\theta}=\frac{(n-1)}{\sqrt{3}(n+2)} .
$$

It is important to note here that the proportionality relation between shear and expansion leads to the positive deceleration parameter $(q)$ with isotropic distribution of DE in LRS Bianchi -I space-time. Since we are looking for a model explaining an expanding Universe with acceleration, so, we assume the anisotropic distribution of DE to ensure the present acceleration of Universe. Thus equations (5), (6) and (16) may be re-written as

$$
\begin{gathered}
2 \frac{\ddot{B}}{B}+\frac{\dot{B}^{2}}{B^{2}}=-\omega^{(m)} \rho^{(m)}-\left(\omega^{(d e)}+\delta\right) \rho^{(d e)}, \\
\frac{\ddot{A}}{A}+\frac{\ddot{B}}{B}+\frac{\dot{A} \dot{B}}{A B}=-\omega^{(m)} \rho^{(m)}-\left(\omega^{(d e)}+\gamma\right) \rho^{(d e)}, \\
\dot{\rho}^{(d e)}+3 \rho^{(d e)}\left(1+\omega^{(d e)}\right) H+\rho^{(d e)}\left(\delta H_{x}+2 \gamma H_{y}\right)=0 .
\end{gathered}
$$

The third term of equation (33) arises due to the deviation from $\omega^{(d e)}$ while the first and second terms of equation (33) are deviation free part of $T_{j}^{(d e) i}$. According to equation (33), the behavior of $\rho^{(d e)}$ is controlled by the deviation free part of of EoS parameter of DE but deviation will affect $\rho^{(d e)}$ indirectly, since as can be seen later, they affect the value of EoS parameter. But we are looking for physically viable models of Universe consistent with observations. Hence we constrained $\delta(t)$ and $\gamma(t)$ by assuming the special dynamics which is consistent with (33). The dynamics of skewness parameter on $\mathrm{x}$-axis $(\delta)$ and $\mathrm{y}$-axis or z-axis $(\gamma)$ are given by

$$
\delta=-\frac{2 m H H_{y}}{\rho^{(d e)}}
$$




$$
\gamma=\frac{m H H_{x}}{\rho^{(d e)}}
$$

where $m$ is the dimensionless constant that parameterizes the amplitude of the deviation from $\omega^{(d e)}$ and can be given real values.

Now, subtracting equation (32) from (33), we get

$$
\frac{\ddot{B}}{B}-\frac{\ddot{A}}{A}+\frac{\dot{B}^{2}}{B^{2}}-\frac{\dot{A} \dot{B}}{A B}=(\gamma-\delta) \rho^{(d e)},
$$

Using equations (91), (18), (34) and (35), from (36) we obtain

$$
\frac{\ddot{B}}{B}+\left[\frac{3\left(n^{2}-1\right)+m(n+2)^{2}}{3(n-1)}\right] \frac{\dot{B}^{2}}{B^{2}}=0,
$$

The general solution of equation (37) has the form

$$
B=\left(k_{1} t+k_{0}\right)^{\frac{3(n-1)}{n_{1}}},
$$

where $n_{1}=3 N_{1}+m(n+2)^{2}$ with $N_{1}=(n-1)(n+2)$.

For $A$ in this case we find

$$
A=\left(k_{1} t+k_{0}\right)^{\frac{3 n(n-1)}{n_{1}}} .
$$

It is important to note here that we obtain power law solution by assuming proportionality relation between shear scalar $(\sigma)$ and expansion $(\theta)$ which seems to describe the dynamics of Universe from big bang to present epoch while a series of works: Yadav and Yadav (2011); Yadav et al (2011); Amirhashchi et al (2011); Kumar and Yadav (2011); Akarsu and Kilinc (2010) and recently Yadav (2011) have obtained the power law solution by assuming special law of variation of Hubble's parameter. So, we represent the new features of power law expansion. In this paper, we show how $\sigma \propto \theta$ model with metric (1) behaves in presence of perfect fluid and anisotropic DE components.

Now, the metric (11) reduces to

$$
d s^{2}=-d t^{2}+\left(k_{1} t+k_{0}\right)^{\frac{6 n(n-1)}{n_{1}}} d x^{2}+\left(k_{1} t+k_{0}\right)^{\frac{6(n-1)}{n_{1}}}\left(d y^{2}+d z^{2}\right) .
$$

In view of the assumption $\omega^{(m)}=$ const., equation (15) can be integrated to obtain

$$
\rho^{(m)}=\rho_{0} a^{-3\left(\omega^{(m)}+1\right)},
$$

where $\rho_{0}$ is the positive constant of integration.

The physical parameter such as directional Hubble parameter $\left(H_{x}, H_{y}\right.$ or $\left.H_{z}\right)$, average Hubble parameter $(H)$, anisotropy parameter $\left(A_{m}\right)$, shear scalar $(\sigma)$, expansion scalar $(\theta)$ and spatial volume $(V)$ of model (40) are respectively given by

$$
\begin{gathered}
H_{x}=\frac{3 n(n-1)}{n_{1}} \frac{k_{1}}{\left(k_{1} t+k_{0}\right)}, \\
H_{y}=H_{z}=\frac{3(n-1)}{n_{1}} \frac{k_{1}}{\left(k_{1} t+k_{0}\right)}, \\
H=\frac{N_{1}}{n_{1}} \frac{k_{1}}{\left(k_{1} t+k_{0}\right)}, \\
A_{m}=\frac{2(n-1)^{2}}{(n+2)^{2}}, \\
\sigma^{2}=\frac{3(n-1)^{4} k_{1}^{2}}{n_{1}^{2}\left(k_{1} t+k_{0}\right)^{2}}, \\
\theta=\frac{3 N_{1}}{n_{1}} \frac{k_{1}}{\left(k_{1} t+k_{0}\right)}, \\
V=\left(k_{1} t+k_{0}\right)^{\left(3 N_{1} / n_{1}\right)} .
\end{gathered}
$$




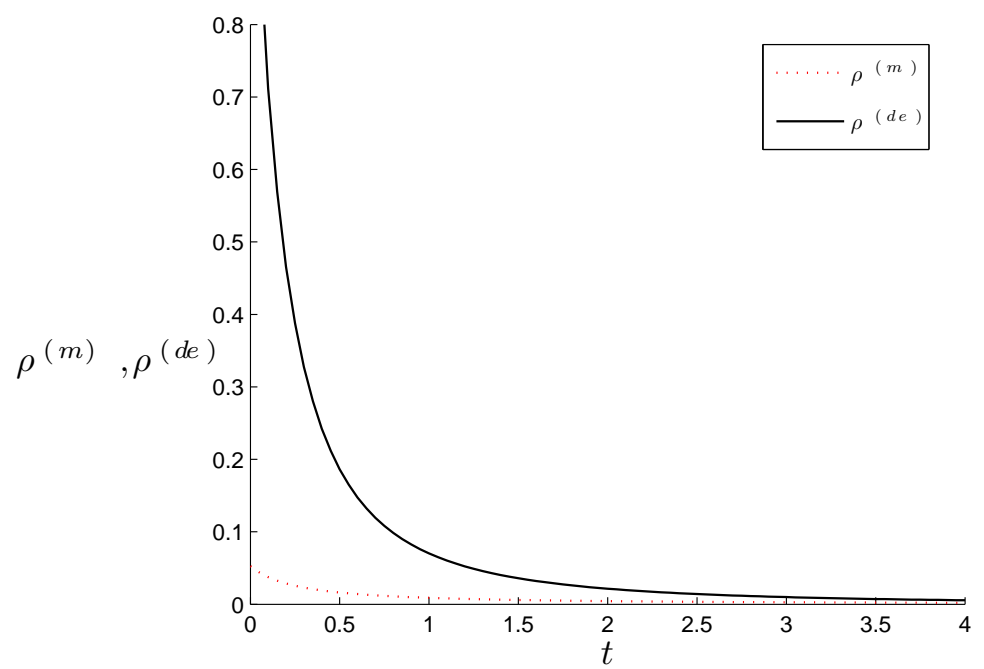

Figure 1: Plot of matter density $\left(\rho^{(m)}\right)$ and DE density $\left(\rho^{(d e)}\right)$ versus time.

The average scale factor $(a)$ and DP $(q)$ are found to be

$$
\begin{gathered}
a=\left(k_{1} t+k_{0}\right)^{\left(N_{1} / n_{1}\right)}, \\
q=\frac{n_{1}}{N_{1}}-1=2+\frac{m}{N_{1}}(n+2)^{2} .
\end{gathered}
$$

As one sees, the DP $q$ is a constant. The sign of $q$ indicates whether the model inflates or not. A positive sign of $q$, i. e. $n_{1} / N_{1}>1$ corresponds to standard decelerating model whereas negative sign of $q$, i. e. $0<n_{1} / N_{1}<1$ indicates acceleration. The recent observations SN Ia, reveal that the present Universe is accelerating and the value of DP lies somewhere in the range $-1<q<0$. It follows that in the derived model, one can choose the value of DP consistent with observations.

From (41) we than find the energy density of perfect fluid

$$
\rho^{(m)}=\rho_{0}\left(k_{1} t+k_{0}\right)^{-3\left(\omega^{(m)}+1\right) N_{1} / n_{1}},
$$

on account of (51) from (7) we obtain the dark energy density as

$$
\rho^{(d e)}=\frac{9(2 n+1)(n-1)^{2} k_{1}^{2}}{n_{1}^{2}\left(k_{1} t+k_{0}\right)^{2}}-\frac{\rho_{0}}{\left(k_{1} t+k_{0}\right)^{3\left(\omega^{m}+1\right) N_{1} / n_{1}}},
$$

Now from (34) and (35) skewness parameter are obtained as

$$
\begin{gathered}
\delta(t)=-\frac{6 m(n-1) N_{1} k_{1}^{2}}{9(2 n+1)(n-1)^{2} k_{1}^{2}-\rho_{0} n_{1}^{2}\left(k_{1} t+k_{0}\right)^{2-3\left(\omega^{(m)}+1\right) N_{1} / n_{1}}}, \\
\gamma(t)=\frac{3 m n(n-1) N_{1} k_{1}^{2}}{9(2 n+1)(n-1)^{2} k_{1}^{2}-\rho_{0} n_{1}^{2}\left(k_{1} t+k_{0}\right)^{2-3\left(\omega^{(m)}+1\right) N_{1} / n_{1}}},
\end{gathered}
$$

The EoS parameter of $\mathrm{DE}$ is given by

$$
\omega^{(d e)}=-\frac{\frac{\omega^{(m)} \rho_{0}}{\left.\left(k_{1} t+k_{0}\right)^{3(\omega(m)}+1\right) N_{1} / n_{1}}+\frac{3(n-1)^{2} k_{1}^{2}\left(6-2 m(n+2)-n_{1} /(n-1)\right)}{n_{1}^{2}\left(k_{1} t+k_{0}\right)^{2}}}{\frac{9(2 n+1)(n-1)^{2} k_{1}^{2}}{n_{1}^{2}\left(k_{1} t+k_{0}\right)^{2}}-\frac{\rho_{0}}{\left(k_{1} t+k_{0}\right)^{3\left(\omega^{m}+1\right) N_{1} / n_{1}}}},
$$

It is observed that at $t=-\frac{k_{0}}{k_{1}}$, the spatial volume vanishes while all other parameters diverge. Thus the derived model starts expanding with big bang singularity at $t=-\frac{k_{0}}{k_{1}}$ which can be shifted to $t=0$ by choosing 


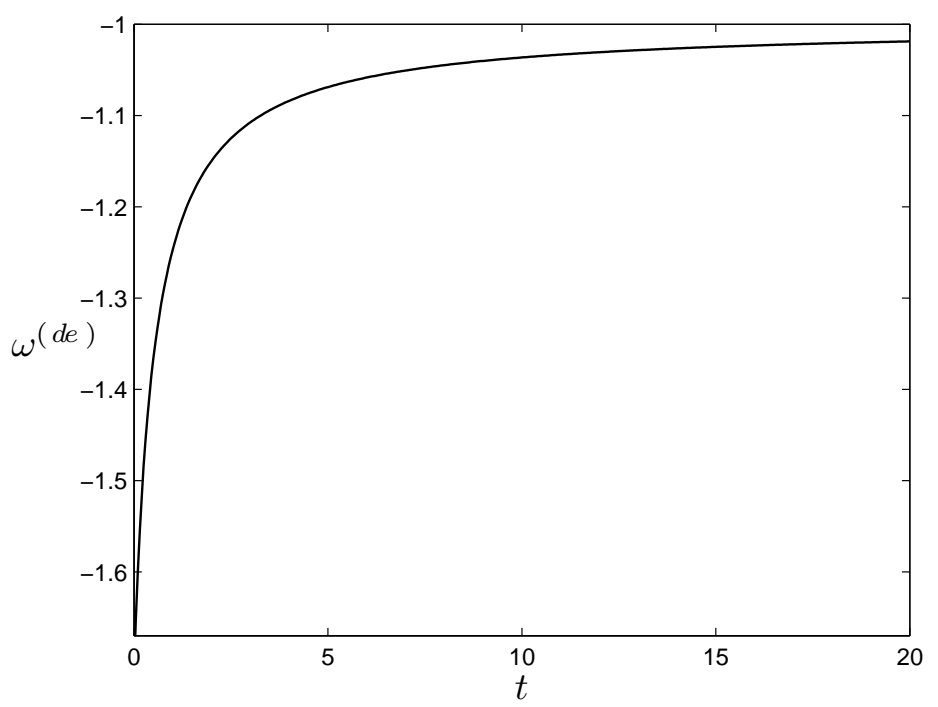

Figure 2: Plot of EoS parameter of DE components $\left(\omega^{(d e)}\right)$ versus time.

$k_{0}=0$. This singularity is point type because the directional scale factors $A(t)$ and $(B(t))$ vanish at initial moment. From Fig. 1, we observe that $\rho^{(m)}$ as well as $\rho^{(d e)}$ remains positive during the cosmic evolution. Therefore the weak energy condition (WEC) as well as null energy condition (NEC) are obeyed in the derived model. Further $\rho^{(m)}$ and $\rho^{(d e)}$ decrease with time, and approach to a small positive values at the present epoch. The parameter $H, \sigma$ and $\theta$ start off with extremely large values and continue to decrease with expansion of universe. Fig. 2 clearly shows that $\omega^{(d e)}$ evolves with negative and it's range is in nice agreement with large scale structure data (Komatsu et al 2009).

The density parameters of perfect fluid and DE are as follows:

$$
\begin{gathered}
\Omega^{(m)}=\frac{\rho_{0} n_{1}^{2}}{3 N_{1}^{2} k_{1}^{2}}\left(k_{1} t+k_{0}\right)^{2-3\left(\omega^{(m)}+1\right) N_{1} / n_{1}} \\
\Omega^{(d e)}=\frac{3(2 n+1)}{(n+2)^{2}}-\frac{\rho_{0} n_{1}^{2}}{3 N_{1}^{2} k_{1}^{2}}\left(k_{1} t+k_{0}\right)^{2-3\left(\omega^{(m)}+1\right) N_{1} / n_{1}}
\end{gathered}
$$

Adding equation (56) and (57), the overall density parameter $(\Omega)$ is obtained as

$$
\Omega=\Omega^{(m)}+\Omega^{(d e)}=\frac{3(2 n+1)}{2(n-1)^{2}} A_{m}
$$

This shows that the overall density parameter $(\Omega)$ depends on the anisotropy parameter $\left(A_{m}\right)$. Fig. 3 demonstrates the behavior of density parameters in the evolution of Universe with appropriate choice of constants of integration and other physical parameters using reasonably well known situations. We observe that initially the ordinary matter density dominates the Universe. But later on, the DE density dominates the evolution which is probably responsible for the accelerated expansion of present-day Universe.

\section{The statefinder \& Distance Modulus Curves}

Sahni et al (2003) proposed a cosmological diagnostic pair $\{r, s\}$ called state finder, which is defined as

$$
\begin{gathered}
r=\frac{\dddot{a}}{a H^{3}}=\left(1-\frac{n_{1}}{N_{1}}\right)\left(1-\frac{2 n_{1}}{N_{1}}\right), \\
s=\frac{r-1}{3\left(q-\frac{1}{2}\right)}=\frac{\left(1-\frac{n_{1}}{N_{1}}\right)\left(1-\frac{2 n_{1}}{N_{1}}\right)-1}{3\left(\frac{n_{1}}{N_{1}}-\frac{3}{2}\right)} .
\end{gathered}
$$




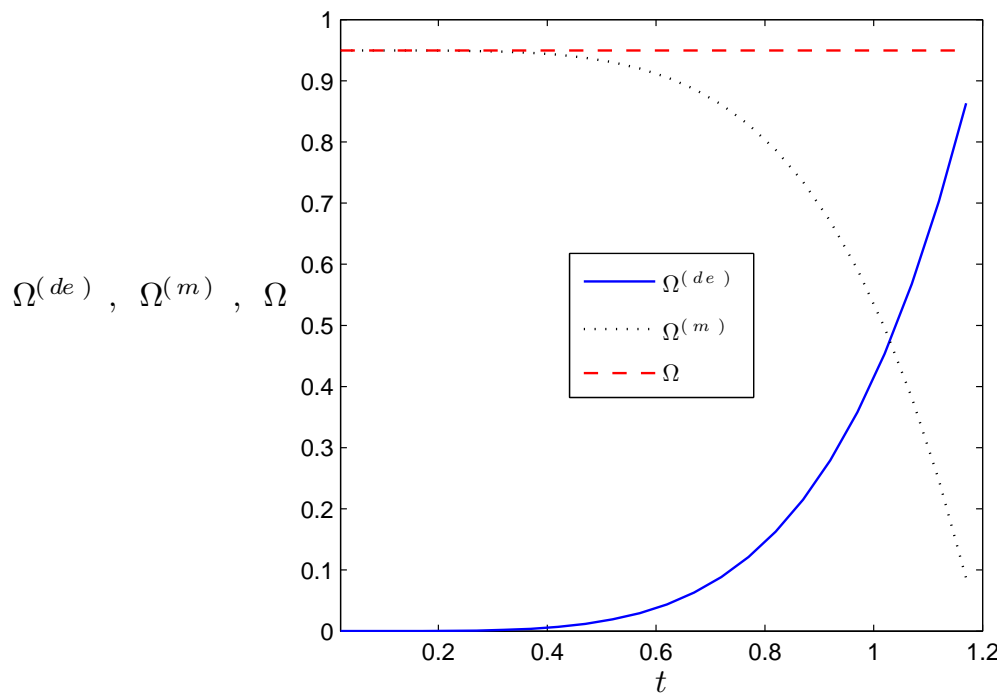

Figure 3: Plot of density parameters versus time.

The dynamics of statefinder $\{r, s\}$ depends on constant $n_{1}$ and $N_{1}$. It follows that in derived model, one can choose the pair of statefinder which can successfully differentiate between a wide variety of DE models including cosmological constant, quintessence, phantom, quintom, the chaplygin gas, braneworld models and interacting DE models. For example if we put $n_{1}=0$, the statefinder pair will be $\{1,0\}$ which yields the $\Lambda C D M(\operatorname{cosmolog}$ ical constant cold dark matter) model. The statefinder diagnosis for holographic DE model in non flat Universe has been analyzed by Setare et al (2007).

The distance modulus is given by

$$
\mu=5 \log d_{L}+25,
$$

where the luminosity distance $d_{L}$ is defined as

$$
d_{L}=r_{1}(1+z) a_{0},
$$

where $z$ and $a_{0}$ represent red shift parameter and present scale factor respectively.

Let us now assume that $T=k_{1} t+k_{0}$. Thus equation (49) may be rewritten as

$$
a=T^{n_{2}},
$$

where $n_{2}=N_{1} / n_{1}$

For determination of $r_{1}$, we assume that a photon emitted by a source with co-ordinate $r=r_{1}$ and $T=T_{1}$ and received at a time $T_{0}$ by an observer located at $r=0$. Then we determine $r_{1}$ from

$$
r_{1}=\int_{T_{1}}^{T_{0}} \frac{d T}{a} .
$$

Solving equations (61) - (64), one can easily obtain the expression for distance modulus $(\mu)$ in term of red shift parameter $(z)$ as

$$
\mu=5 \log \left[\frac{n_{2} k_{1}}{H_{0}\left(1-n_{2}\right)(1+z)^{\frac{1-2 n_{2}}{n_{2}}}}\left((1+z)^{\frac{1-n_{2}}{n_{2}}}-1\right)\right]+25 .
$$

The comparison between the derived model and SNLS type Ia supernovae data can be seen in Fig.4. The dotted line represents the observed distance modulus by SNLS type Ia supernovae data where as solid line represents the analyzed distance modulus $\mu$ of the derived model. It is observed that the derived model is best fit with high redshift values. 


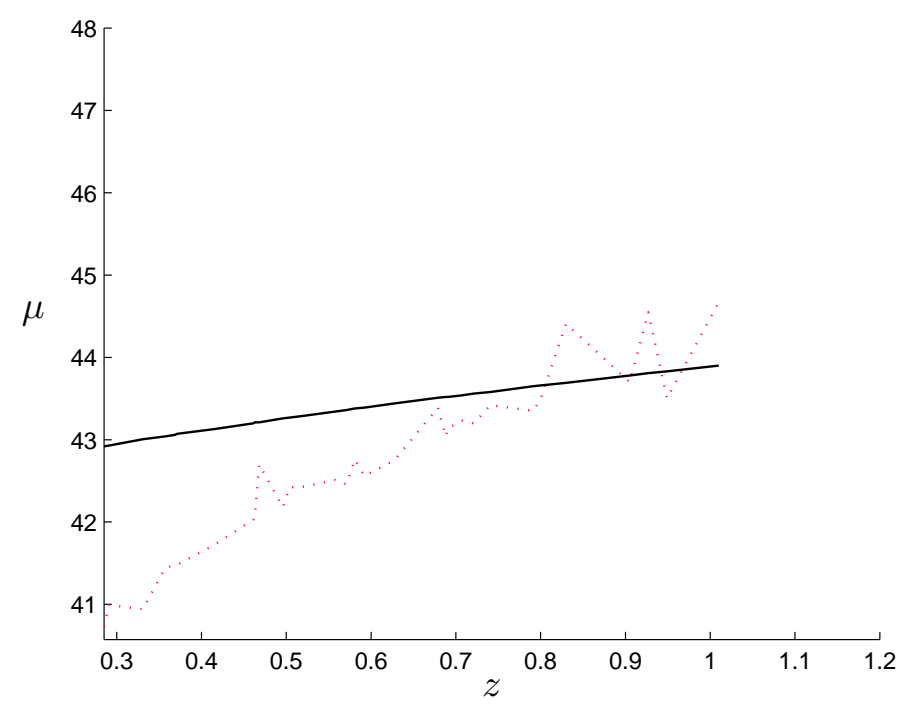

Figure 4: Distance modulus as a function of the redshift to the derived model compared with SNLS type Ia supernovae data from Astier et al. (2006)

\section{Conclusion}

In this paper, we have studied a spatially homogeneous and anisotropic LRS Bianchi-I space time filled with perfect fluid and anisotropic DE possessing dynamical energy density. Studying the interaction between the ordinary matter and DE will open up the possibility of detecting DE. It should be pointed out that evidence was recently provided by Abell-Cluster A586 in support of interaction between DE and dark matter (Bertolami et al 2007; Le Delliou et al 2007). Let us now concentrate on some other works on minimally interacting perfect fluid and DE models of Universe, especially the works by Akarsu and Kilinc (2010) and Yadav (2011). In both the works, the field equations have been solved by using special law of variation of Hubble's parameter which yields the constant value of DP where as the present investigation is one with power law solution by taking into account the proportionality relation between shear scalar $(\sigma)$ and expansion scalar $(\theta)$. It is to be noted that our procedure of solving the field equations is altogether different from what Akarsu and Kilinc (2010) have adapted in LRS Bianchi-I space-time. However, a common feature of all these power law solution models is to describe the dynamics of Universe from big bang to present epoch.

In the derived model, the EoS parameter of $\mathrm{DE}\left(\omega^{(d e)}\right)$ is obtained as time varying and it is evolving with negative sign which may be attributed to the current accelerated expansion of Universe. Also note that the isotropic distribution of DE is not possible in LRS Bianchi Type I space-time because the isotropic distribution of $\mathrm{DE}$ leads to the positive value of DP which can not explain the current accelerated expansion of Universe while for anisotropic distribution of DE, DP evolves with negative sign. The distance modulus curve of derived model is in good agreement with SNLS type Ia supernovae for high redshift value which in turn imply that the derived model is physically realistic.

The age of Universe is given by

$$
T_{0}=\frac{N_{1}}{n_{1}} H_{0}^{-1}-\frac{k_{0}}{k_{1}}
$$

which is different from the present estimate i.e. $T_{0}=H_{0}^{-1}=14 G y r$. But if we take $k_{0}=0$ and $N_{1} / n_{1}=1$, i.e., $m=2(1-n) /(2+n)$, where $m$ is the constant describing the anisotropy of DE and $n$ is the constant giving the proportionality condition between shear and expansion scalar, then the derived model is in good agreement with the present age of Universe. 


\section{Acknowledgements}

The authors would like to thank the anonymous referee for his/her useful comments to improve this work and drawing our attention to a couple of references relevant to our studies. Author (AKY) is thankful to The

Institute of Mathematical Science (IMSc), Chennai, India for providing facility and support where part of this work was carried out.

\section{References}

[1] Akarsu, O., Kilinc, C. B.: Gen. Relativ. Grav. 42, 119 (2010)

[2] Amirhashchi, H., Pradhan, A. and Saha, B.: 333, 295 (2011)

[3] Astier, P. et al.: Astron. Astrophys. 447, 31 (2006)

[4] Benett C. L. et al : Astrophys. J. 464, L1 (1996).

[5] Bertolami, O., Gil Pedro, F. and Le Delliou, M: Phys. Lett. B 654, 165 (2007)

[6] Cai, Y. F., Saridakis, E. N., Setare, M. R. and Xia, J. Q.: Phys. Rept. 493, 1 (2010)

[7] Caldwell, R. R.: Phys. Lett. B 545, 23 (2002)

[8] Caldwell, R.R., Komp, W., Parker, L. and Vanzella, D.A.T.: Phys. Rev. D 73, 023513 (2006)

[9] Chimento, L. P., Jakubi, A. S., Mendez, W. and Maartens, R.: Class. Quant. Grav. 14, 3363 (1997).

[10] Hinshaw et al: Astrophys. J. Suppl. 148, 135 (2003)

[11] Huang, W.: J. Math. Phys. 31, 1456 (1990)

[12] Knop, R.A., et al.: Astrophys. J. 598, 102 (2003)

[13] Koivisto, T. and Mota, D. F.: (2008a) arXiv:0801.3676 [astro-ph]

[14] Koivisto, T. and Mota, D. F.: Astrophys. J. 679, 1 (2008b)

[15] Komatsu, E. et al.: Astrophys. J. Suppl. Ser. 180, 330 (2009)

[16] Kumar, S. and Yadav, A. K.: Mod. Phys. Lett. A 26, 647 (2011).

[17] Le Delliou, M., Bertolami, O. and Gil Pedro, F.: AIP Conf. Proc. 957, 421 (2007)

[18] Lima, J. A. S. and Trodden, M.: Phys. Rev. D 53, 4280 (1996).

[19] Lima, J. A. S. and Maia, J. M. F.: Phys. Rev. D 49, 5579 (1994).

[20] Martins, C.J.A.P.: Phil.Trans. Roy. Soc. Lond. A 360, 2681 (2002)

[21] Melchiorri, A., Mersimi, L., Odman, C. J., Trodden, M.: Phys. Rev. D 68, 043509 (2003).

[22] Perlmutter, S. et al.: Astrophys. J. 517, (1999), 565 (1999)

[23] Pradhan, A. and Singh, S. K.: Int. J. Mod. Phys. D 13, 503 (2004).

[24] Pradhan, A. and Pandey, P.: Astrophys. Space Sci. 301, 221 (2006).

[25] Riess, A. G. et al.: Astron. J. 116, 1009 (1998) (1998)

[26] Saha, B.: Astrophys. Space Sci. 302, 83 (2006a).

[27] Saha, B.: Int. J. Theor. Phys. 45, 983 (2006b).

[28] Saha, B.: Chin. J. Phys. 13, 1035 (2005).

[29] Sahni, V., Saini, T. D., Starbinsky, A. A., Alam, U.: JETP lett. 77, 201 (2003) 
[30] Smoot, G. F. et al: Astrophys. J. 396, L1 (1992)

[31] Spregel, D. N. et al: Astrophys. J. Suppl. 170, 377 (2007)

[32] Setare, M. R., Zhang, J. and Zhang, X.: JCAP 0703, 007 (2007)

[33] Setare, M. R.: Eur. Phys. J. C 50, 991 (2007)

[34] Setare, M. R. and Saridakis, E. N.: Phys. Lett. B 668, 177 (2008)

[35] Setare, M. R. and Saridakis, E. N.: JCAP 0903, 002 (2009a)

[36] Setare, M. R. and Saridakis, E. N.: Int. J. Mod. Phys. D 18, 549 (2009b)

[37] Setare, M. R.: Phys. Lett. B 641, 130 (2006)

[38] Steinhardt, P. J., Wang, L. M. and Zlatev, I.: Phys. Rev. D 59, 123504 (1999)

[39] Yadav, A. K., Yadav, L.:Int. J. Theor. Phys. 50, 218 (2011); arXiv:1007.1411 [gr-qc].

[40] Yadav, A. K., Rahaman, F., Ray, S.: Int. J. Theor. Phys. 50, 871 (2011).

[41] Yadav, A. K.: Astrophys. Space Sc. DOI: 10.1007/s10509-011-0745-3 (2011) 